\title{
Anisotropic Polar Magneto-Optic Kerr Effect of Ultrathin Fe/GaAs(001) Layers due to Interfacial Spin-Orbit Interaction
}

\author{
M. Buchner, ${ }^{1}$ P. Högl, ${ }^{2}$ S. Putz, ${ }^{2}$ M. Gmitra, ${ }^{2}$ S. Günther, ${ }^{3}$ M. A. W. Schoen, ${ }^{1}$ M. Kronseder, ${ }^{1}$ \\ D. Schuh, ${ }^{1}$ D. Bougeard, ${ }^{1}$ J. Fabian, ${ }^{2}$ and C. H. Back ${ }^{1}$ \\ ${ }^{1}$ Institute of Experimental and Applied Physics, University of Regensburg, Regensburg 93040, Germany \\ ${ }^{2}$ Institute of Theoretical Physics, University of Regensburg, Regensburg 93040, Germany \\ ${ }^{3}$ Department of Materials, ETH Zürich, Zürich 8093, Switzerland
}

(Received 22 January 2016; revised manuscript received 30 June 2016; published 6 October 2016)

\begin{abstract}
We report the observation of the anisotropic polar magneto-optical Kerr effect in thin layers of epitaxial $\mathrm{Fe} / \mathrm{GaAs}(001)$ at room temperature. A clear twofold symmetry of the Kerr rotation angle depending on the orientation of the linear polarization of the probing laser beam with respect to the crystallographic directions of the sample is detected for ultrathin magnetic films saturated out of the film plane. The amplitude of the anisotropy decreases with increasing Fe film thickness, suggesting that the interfacial region is the origin of the anisotropy. The twofold symmetry is fully reproduced by model calculations based on an interference of interfacial Bychkov-Rashba and Dresselhaus spin-orbit coupling.
\end{abstract}

DOI: 10.1103/PhysRevLett.117.157202

Spin-orbit coupling (SOC) plays an important role in solid-state systems and can lead to emerging spin-orbit fields (SOFs) at interfaces that inherently lack inversion symmetry. The reduced symmetry at heterostructure interfaces or surfaces leads to electron momentum dependent SOFs, which affect the electronic properties of the material $[1,2]$. Interfacial SOFs result in a plethora of novel effects such as tunneling anisotropic magnetoresistance (TAMR) [3-6], anisotropic crystalline anisotropic magnetoresistance (CAMR) [7], current driven torques at interfaces [8-12], the formation of skyrmion lattices [13], or the emergence of topological superconductors [14]. So far most investigations of the interfacial anisotropies caused by SOFs rely on transport measurements on structured samples. This Letter reports on an optical detection of the spin-orbit-based crystalline magneto-optical anisotropy for a frequently used benchmark ferromagnet-semiconductor interface, that of $\mathrm{Fe} / \mathrm{GaAs}$. Our optical approach allows detection of a $C_{2 v}$ symmetry in the magneto-optical signal, which is connected to the interfacial SOC, even at room temperature, making it an ideal tool to characterize spin-orbit related effects in a noninvasive way on full film samples.

Recently, density functional theory (DFT) calculations have proposed that SOFs at the $\mathrm{Fe} / \mathrm{GaAs}(001)$ interface should give rise to anisotropic optical properties [15]. The $C_{2 v}$ symmetry of the interface is predicted to enter the optical conductivity tensor and should hence lead to an anisotropic polar magneto-optical Kerr effect (AP-MOKE). This means that the Kerr rotation angle $\theta_{K}$ and Kerr ellipticity $\varepsilon_{K}$ in a polar magneto-optical Kerr effect (P-MOKE) configuration depend on the angle between the linear polarization of the probing laser beam and the crystallographic axes of the sample, reflecting the twofold symmetry of the interface.
Here, we show that an AP-MOKE can indeed be observed in ultrathin $\mathrm{Fe} / \mathrm{GaAs}(001)$ at room temperature. The Kerr rotation angle is significantly larger with the laser being polarized along the [110] crystallographic axis, compared to a polarization in the [11 10$]$ direction, altogether showing a uniaxial symmetry. The $C_{2 v}$ symmetry of the effect can be described by a theoretical model based on the interference of Bychkov-Rashba [16,17] and Dresselhaus [18] SOC. For larger Fe film thicknesses and polycrystalline films the $C_{2 v}$ symmetry vanishes as expected from theory. Note that the observed $C_{2 v}$ symmetry of the magnetooptical response reflects the anisotropy of the optical conductivity tensor, which contains all allowed optical transitions [15], whereas the Boltzmann dc conductivity of a two-dimensional electron gas with Bychkov-Rashba and Dresselhaus SOC shows a vanishing anisotropy [19].

In the experiments reported here, we investigate 4, 6, 8, and 20 monolayer (ML) thick Fe films, which are grown by MBE onto a $100 \mathrm{~nm}$ thick buffer layer of undoped GaAs. The latter is grown to smoothen the underlying (001)oriented GaAs substrate prior to the $\mathrm{Fe}$ growth. The epitaxial growth and the thickness of the Fe layer are monitored in situ by recording RHEED. Subsequently, to protect the Fe layers from oxidation they are capped in situ with a $10 \mathrm{~nm}$ thick $\mathrm{MgO}$ layer and a $6 \mathrm{~nm}$ thick layer of $\mathrm{Al}_{2} \mathrm{O}_{3}$.

The P-MOKE measurements are carried out at room temperature using the setup shown in Fig. 1(a). A diode laser with a wavelength of $405 \mathrm{~nm}$ is used as the light source. This wavelength is chosen according to the DFT calculations of Ref. [15], as the theoretical considerations suggest a strong AP-MOKE for this particular photon energy [20]. The light is linearly polarized by a polarizer $P_{1}$ and guided to the sample via the two mirrors $M_{1}$ and $M_{2}$. 
The sample is mounted on a special sample holder, which enables a rotation of the sample about its [001] crystallographic direction, which is parallel to the magnetic field and the direction of the incident laser beam. Therefore, it is possible to vary the angle between the in-plane crystallographic directions of the sample and the fixed linear polarization direction of the incident laser beam. Here, it is extremely important to ensure that the sample is properly mounted on the sample holder and that the laser hits the sample perfectly at normal incidence. Even a small misalignment between the sample normal, the rotation axis, and the direction of the incident laser beam causes a deviation of the back reflected beam from the desired optical path by several degrees as soon as the sample is rotated. Such deviations can lead to spurious signal signatures in the angle dependent measurements and thus have to be avoided.

Because of the P-MOKE the reflected light undergoes a change of its linear polarization to a rotated, elliptical polarization state, characterized by the Kerr rotation angle $\theta_{K}$ and Kerr ellipticity $\varepsilon_{K}$. We use two different measurement schemes for the detection of the Kerr effect [see Fig. 1(a)]. The first one uses a Wollaston prism (WP) that splits the light into two orthogonally polarized beams whose intensities $I_{1}$ and $I_{2}$ are measured with a balanced photodiode detector. The detector transmits both the sum signal
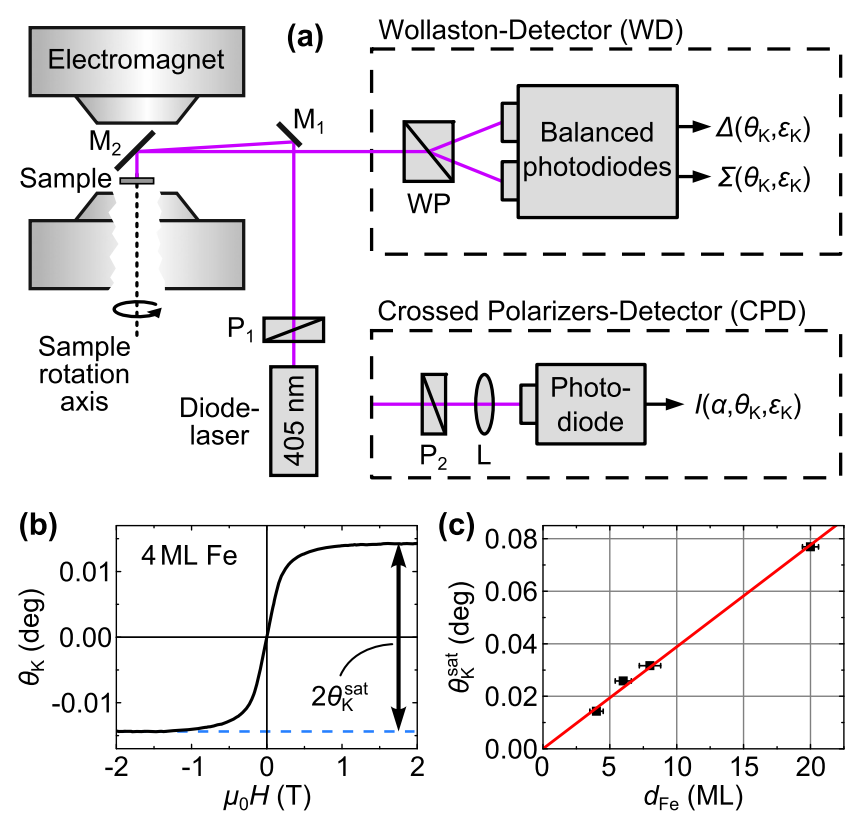

FIG. 1. (a) Sketch of the P-MOKE setup. The sample is mounted on a rotatable sample holder within the field of an electromagnet. The Kerr signal of the sample can be measured via a Wollaston-detector unit or via a crossed polarizer detector. (b) P-MOKE loop of $4 \mathrm{ML} \mathrm{Fe/GaAs(001)} \mathrm{measured} \mathrm{with} \mathrm{the}$ Wollaston-detector. The height of the loop in saturation, $2 \theta_{K}^{\text {sat }}$, is used as a measure for the strength of the P-MOKE. (c) Kerr rotation angle $\theta_{K}^{\text {sat }}$ as a function of the Fe layer thickness. The solid line is a linear fit.
$\Sigma\left(\theta_{K}, \varepsilon_{K}\right)=I_{1}+I_{2}$ and the difference signal $\Delta\left(\theta_{K}, \varepsilon_{K}\right)=$ $I_{1}-I_{2}$ of the two single photodiodes. A theoretical analysis of the system using Stokes vectors and Müller matrices shows that $\Sigma\left(\theta_{K}, \varepsilon_{K}\right)=I_{0}$ and $\Delta\left(\theta_{K}, \varepsilon_{K}\right)=$ $I_{0} \sin \left(2 \theta_{K}\right) \cos \left(2 \varepsilon_{K}\right)$, where $I_{0}$ is the light intensity right at the sample position [20]. Hence, using the smallangle approximation, one finds the following expression for the Kerr rotation angle: $\theta_{K} \approx \Delta\left(\theta_{K}, \varepsilon_{K}\right) / 2 \Sigma\left(\theta_{K}, \varepsilon_{K}\right)$. Therefore, the Wollaston-detector (WD) method yields absolute values for the Kerr rotation angle and separates any signal contributions originating from the Kerr ellipticity. Figure 1(b) displays an out-of-plane, hard axis loop of the $4 \mathrm{ML} \mathrm{Fe} / \mathrm{GaAs}$ sample, measured with the WD. The sample exhibits a maximum Kerr rotation of approximately $\pm 0.0145^{\circ}$ in saturation. For the AP-MOKE measurements we use the height of the magnetization loops in saturation, $2 \theta_{K}^{\text {sat }}$, as a measure for the strength of the polar magnetooptical Kerr effect. Figure 1(c) illustrates the Fe layer thickness dependence of $\theta_{K}^{\text {sat }}$. As the penetration depth of the laser lies well above the thickness of the investigated Fe layers, the Kerr rotation shows a linear increase with film thickness.

The second measurement method used in the experiment utilizes a crossed polarizer detector (CPD) scheme [see Fig. 1(a)]. Here, a second linear polarizer $P_{2}$ is used to probe the polarization state of the reflected, Kerr rotated light. The optical axis of $P_{2}$ deviates slightly from the perfectly crossed state. The transmitted light is focused with the aid of a lens $\mathrm{L}$ onto a single photodiode, which detects the light intensity $I\left(\alpha, \theta_{K}, \varepsilon_{K}\right)$, where $\alpha$ is the angle of $P_{2}$ with respect to the perfectly crossed state. A theoretical analysis of the setup shows that $I$ is given by $I\left(\alpha, \theta_{K}, \varepsilon_{K}\right)=$ $I_{0} / 2\left[1-\cos \left(2\left(\alpha-\theta_{K}\right)\right) \cos \left(2 \varepsilon_{K}\right)\right]$ [20]. Hence, in contrast to the WD method, the CPD mixes the $\theta_{K}$ and $\varepsilon_{K}$ signal contributions. However, it can be shown that the Kerr rotation angle is directly proportional to the intensity difference between the positive and negative applied magnetic field, $\Delta I=|I(+\boldsymbol{H})-I(-\boldsymbol{H})|$, resulting in $\theta_{K} \approx \Delta I / 2 I_{0} \sin (2 \alpha)$. The CPD does not allow us to directly determine $I_{0}$ and the angle $\alpha$ is in general an illdefined quantity. Thus, unlike the WD, the CPD gives only a relative measure for the Kerr rotation signal $\theta_{K}$. However, the advantage of the CPD compared to the WD is its better signal-to-noise ratio due to its simpler optical and electrical layout.

In this work we investigate the anisotropy of the polar Kerr rotation angle $\theta_{K}^{\text {sat }}$ with respect to the polarization direction of the incident laser beam. Thin Fe films on GaAs(001) exhibit both a cubic magnetocrystalline symmetry, originating from the bulk symmetry of the material, and a uniaxial magnetocrystalline symmetry, which stems from the $\mathrm{Fe} / \mathrm{GaAs}(001)$ interface. For ultrathin films with a thickness of only a few monolayers the uniaxial anisotropy dominates, leading to a magnetic easy axis along the crystallographic [110] direction [21-26]. Both ferromagnetic 


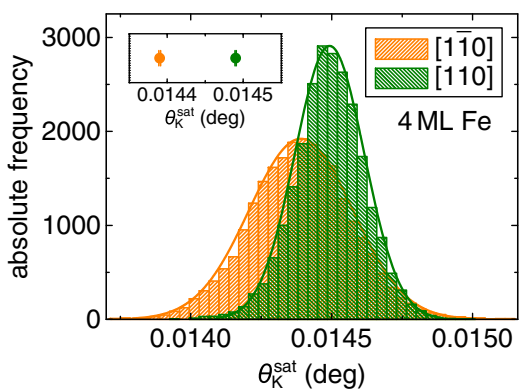

FIG. 2. Histograms of the Kerr rotation angles $\theta_{K}^{\text {sat }}$ for the laser being polarized along the [110] and [110] crystallographic directions for $4 \mathrm{ML} \mathrm{Fe/GaAs.} \mathrm{The} \mathrm{inset} \mathrm{shows} \mathrm{the} \mathrm{mean} \mathrm{values}$ for both polarization directions with the corresponding error bars.

resonance and longitudinal magneto-optical Kerr effect measurements confirm this behavior for the samples investigated here [20].

Figure 2 displays histograms of repeated independent measurements of the absolute value of the Kerr rotation angles $\theta_{K}^{\text {sat }}$ on the $4 \mathrm{ML} \mathrm{Fe} / \mathrm{GaAs}$ sample with the incident laser beam being polarized along the [110] and [110] crystallographic directions, respectively, measured with the WD, see the Supplemental Material [20]. The statistical analysis reveals a clear difference between both polarization directions (see the inset of Fig. 2) with the Kerr rotation angle $\theta_{K}^{\text {sat }}$ in the [110] direction being $\approx 0.7 \%$ larger than in the $[1 \overline{1} 0]$ direction.

In Figs. 3(a)-3(c) we show the anisotropy of the Kerr rotation angle $\theta_{K}^{\text {sat }}$ for 4,6 , and 8 monolayer $\mathrm{Fe} / \mathrm{GaAs}$ measured with the CPD while rotating the sample about the direction of the incident laser beam and keeping both $I_{0}$ and $\alpha$ fixed. The azimuthal angle $\phi$ in the plots indicates the direction of the linear polarization of the incident laser beam with respect to the crystallographic axes of the samples. The data are normalized to the value taken along the hard magnetic direction [11̄0]. In all three cases the measurements reveal a clear twofold symmetry of the Kerr rotation angle with maxima (minima) along the [110] ([110]) directions, the principal axes of the interfacial spin-orbit coupling fields. Note that the amplitude of the signal decreases with increasing Fe film thickness indicating its interfacial origin.

To rule out any systematic errors from the setup as the possible origin for the measured twofold symmetry, we additionally perform polarization angle dependent measurements on a single crystalline $20 \mathrm{ML}$ thick Fe/GaAs sample and a polycrystalline $100 \mathrm{~nm}$ thick permalloy (Py) film grown onto oxidized GaAs. These measurements are carried out in an analogous way to the measurements of Figs. 3(a)-3(c) and are shown in Figs. 3(d)-3(f). In neither of these control experiments do we see a clear twofold behavior of $\theta_{K}^{\text {sat }}$. In the $20 \mathrm{ML} \mathrm{Fe} / \mathrm{GaAs}$ sample the AP-MOKE is - due to its interface character-too weak to be detected and in Py it should be completely absent due
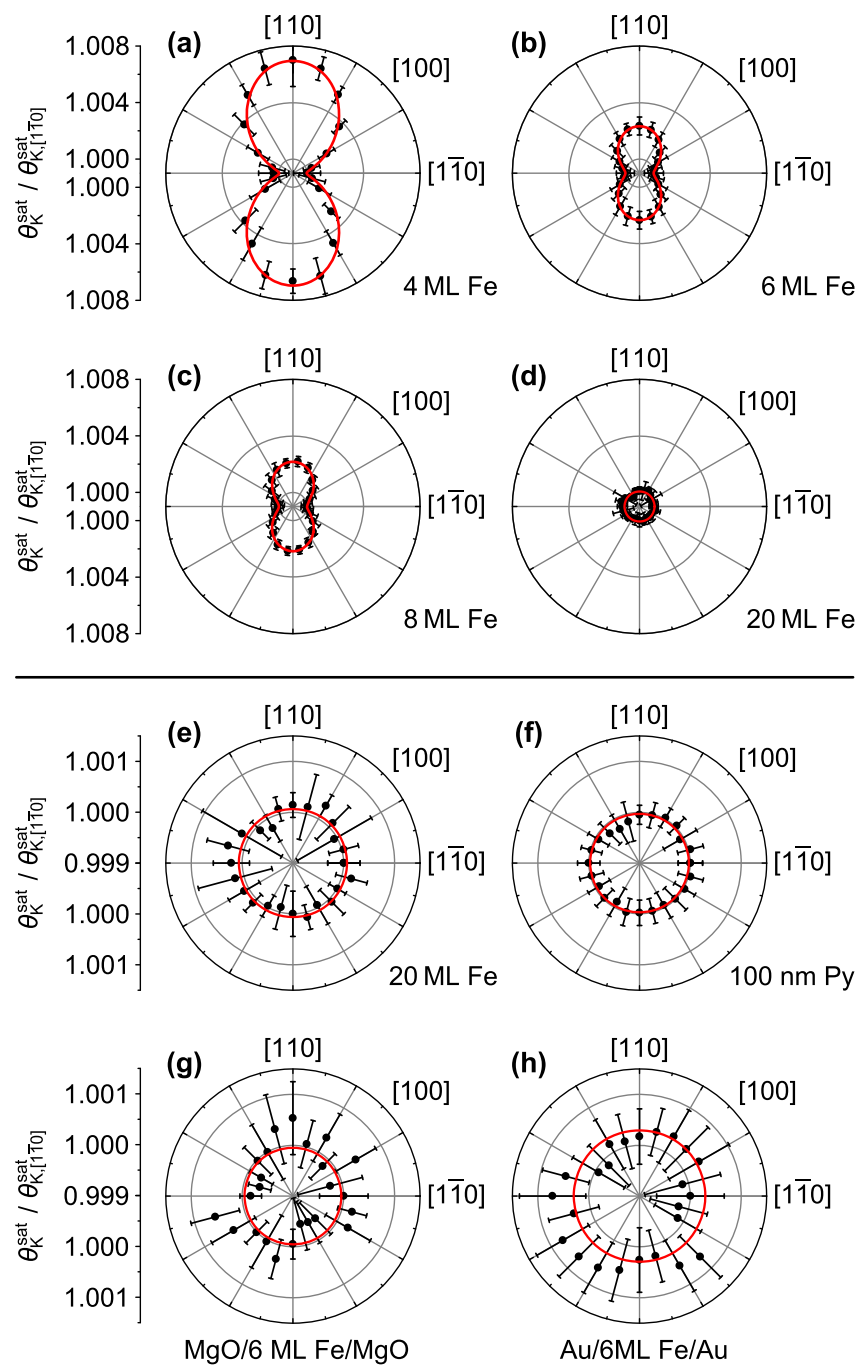

FIG. 3. (a)-(d) Maximum Kerr rotation $\theta_{K}^{\text {sat }}$ normalized to the Kerr rotation along the [1실 direction for different polarization directions of the incident laser beam for 4, 6, 8, and 20 monolayer $\mathrm{Fe} / \mathrm{GaAs}(001)$. The symbols are experimental data. In (a)-(c) the solid lines are fits using the phenomenological model (1). The solid line in (d) represents the average value of the data points. (e) Data of (d) rescaled for better visibility. (f) Angular dependence of the Kerr rotation for a $100 \mathrm{~nm}$ thick film of Py/GaAs. (g),(h) Angular dependence of the Kerr rotation for $3 \mathrm{~nm} \mathrm{Au} / 4 \mathrm{~nm} \mathrm{MgO} / 6 \mathrm{ML} \mathrm{Fe} / \mathrm{MgO}(001)$ and $3 \mathrm{~nm} \mathrm{Au} / 6 \mathrm{ML}$ $\mathrm{Fe} / 100 \mathrm{~nm} \mathrm{Au} / \mathrm{GaAs}(001)$.

to the polycrystallinity of the sample. Moreover, we also find a vanishing Kerr rotation anisotropy for symmetrically grown, single crystalline $\mathrm{MgO} / 6 \mathrm{ML} \mathrm{Fe} / \mathrm{MgO}$ and $\mathrm{Au} / 6 \mathrm{ML} \mathrm{Fe} / \mathrm{Au}$ samples [see Figs. 3(g) and 3(h)], both lacking the interfacial $C_{2 v}$ symmetry of the SOC. Therefore, we link the observations of Figs. 2 and 3(a)-3(c) to the anisotropic polar magneto-optical Kerr effect originating from the interfacial SOC at the Fe/GaAs interface [15].

The observed symmetry of the Kerr rotation angle for the ultrathin Fe layers compares nicely to ab initio calculations based on a 3 ML Fe/9 ML GaAs(001) model system 
$[15,20]$. The theoretical considerations reveal a pronounced uniaxial behavior of $\theta_{K}^{\text {sat }}$ with strong anisotropies in the ultraviolet and violet wavelength range. However, as the DFT calculations assume an ideal $\mathrm{Fe} / \mathrm{GaAs}$ interface at $T=0$, they suggest Kerr rotation anisotropies of the order of $\approx 50 \%$ for a wavelength of $405 \mathrm{~nm}$ [20], in contrast to the actually measured anisotropies, which lie well below $1 \%$ at room temperature. Note that such large discrepancies between $a b$ initio theory and experiment are not unusual and have already been observed previously. In general, spin-orbit related effects become weaker with increasing temperature. For example, the TAMR ratio of $\mathrm{Fe} / \mathrm{GaAs} / \mathrm{Au}$ tunnel junctions, which was shown to be of the order of some tenths of a percent at $4.2 \mathrm{~K}[3,5]$, linearly decreases with increasing temperature and even vanishes for certain bias conditions at room temperature [27]. Moreover, firstprinciple calculations suggest for $\mathrm{Fe}$-based tunnel junctions a large TAMR of up to $35 \%$ [28,29], which is 2 orders of magnitude larger than experimentally observed. So, just like in the case of TAMR, a combination of elevated temperatures and enhanced $a b$ initio values due to the assumption of perfect interfaces can easily accumulate to the observed 2 orders of magnitude difference between experiment and theory.

The experimentally measured twofold symmetry of the AP-MOKE suggests that the effect originates from the SOFs present at the $\mathrm{Fe} / \mathrm{GaAs}$ interface. They exhibit the same twofold symmetry that stems from the combination of Dresselhaus and Bychkov-Rashba spin-orbit interaction arising from the bulk inversion asymmetry of GaAs and the structure inversion asymmetry of the $\mathrm{Fe} / \mathrm{GaAs}$ interface, respectively. Therefore, we consider a phenomenological model that is based on rather general symmetry considerations and was used to explain the angular dependence of TAMR [1,3,6] and CAMR [7] in $\mathrm{Fe} / \mathrm{GaAs}$ based heterostructures. For more details see the Supplemental Material [20]. We take into account the spin-orbit interaction, which can be written as $H_{\mathrm{SOI}} \sim \boldsymbol{w}\left(\boldsymbol{k}_{\|}\right) \cdot \hat{\boldsymbol{\sigma}}$ with the general form of an effective interfacial spin-orbit field $\boldsymbol{w}(\boldsymbol{k})=\left[w_{x}(\boldsymbol{k}), w_{y}(\boldsymbol{k}), 0\right]$ given in an energy range of optical transitions depending on the photon energy of the incoming laser beam. $\hat{\sigma}$ is a vector whose components are the Pauli matrices. The SOF reflects the symmetry of the Fe/GaAs interface; therefore, $|\boldsymbol{w}(\boldsymbol{k})|$ exhibits $C_{2 v}$ symmetry. From first-principle calculations [15] we know that spin-orbit coupling is crucial to observe an anisotropic Kerr rotation and that the rotation depends on the direction of polarization of the incoming light. The orientation of the magnetization is fixed to the [001] direction. So the system has only two distinguished directions for a given $\boldsymbol{k}$, namely, the polarization of the incoming light $\boldsymbol{p}=[\cos (\phi-\pi / 4)$, $\sin (\phi-\pi / 4), 0]$ and $\boldsymbol{w}(\boldsymbol{k})$. The azimuthal angle $\phi$ is measured with respect to the [1ㅣㅣㄹ axis. Thus, we can obtain the anisotropy of a scalar quantity such as the Kerr rotation angle by expanding it in powers of products of $\boldsymbol{p}$ and $\boldsymbol{w}(\boldsymbol{k})$ and averaging over the in-plane momenta $\boldsymbol{k}_{\|}$. The Kerr rotation angle up to second order can then be written as $\theta_{K}^{\text {sat }}(\phi) \approx\left\langle a_{1}^{(0)}(\boldsymbol{k})\right\rangle+\left\langle a_{1}^{(2)}(\boldsymbol{k})|\boldsymbol{w}(\boldsymbol{k})|^{2}\right\rangle+\left\langle a_{2}^{(2)}(\boldsymbol{k})[\boldsymbol{p} \cdot \boldsymbol{w}(\boldsymbol{k})]^{2}\right\rangle$. The expansion coefficients $a_{i}^{(j)}(\boldsymbol{k})$ refer to the system in the absence of spin-orbit coupling so they are independent of $\boldsymbol{p}$ and have the cubic symmetry of the bulk materials. The first order term vanishes since $\boldsymbol{w}(\boldsymbol{k})=-\boldsymbol{w}(-\boldsymbol{k})$. By taking into account that the anisotropic contribution of the Kerr rotation angle is small we can derive the angular dependence of the AP-MOKE

$$
\begin{aligned}
& \operatorname{AP}-\operatorname{MOKE}(\phi) \\
& \qquad=\frac{\theta_{K}^{\text {sat }}(\phi)}{\theta_{K,[110]}^{\text {sat }}}-1 \sim\left\langle a_{2}^{(2)}(\boldsymbol{k}) w_{x}(\boldsymbol{k}) w_{y}(\boldsymbol{k})\right\rangle[1-\cos (2 \phi)] .
\end{aligned}
$$

It exhibits exactly the $C_{2 v}$ symmetric angular dependence that was expected from the experimental observation and is proportional to the product of the $x$ and $y$ component of the SOF. The interference of both Bychkov-Rashba and Dresselhaus SOC in the system is crucial to obtain this result because it ensures the necessary symmetry of the SOFs and is consistent with the reference measurements in systems where at least one of the two spin-orbit fields is missing and consequently the AP-MOKE is zero.

Recently, lateral magnetotransport measurements on identically prepared, epitaxial 4,6 , and 8 monolayer thick $\mathrm{Fe} / \mathrm{GaAs}(001)$ films have revealed a robust magnetoresistance anisotropy depending on the direction of the current flow with respect to the crystallographic directions of the sample [7]. Just like in the present case of the AP-MOKE these transport anisotropies have been explained by the presence of interfacial Bychkov-Rashba and Dresselhaus SOFs. However, note that the optical measurements include all optical transitions at a photon energy of $3 \mathrm{eV}$ available in the Brillouin zone, whereas the lateral magnetotransport experiment of Ref. [7] only involves electrons right at the Fermi edge.

In summary, we have shown that SOC at the $\mathrm{Fe} / \mathrm{GaAs}(001)$ interface not only affects the transport properties of the heterostructure, as shown before, but also gives rise to magneto-optical anisotropies. The experiment clearly reveals a twofold Kerr rotation anisotropy for ultrathin $\mathrm{Fe} / \mathrm{GaAs}$ layers. This anisotropy vanishes in control measurements on thicker films or samples without the $C_{2 v}$ symmetry of the SOFs, demonstrating that the observed AP-MOKE stems from the $\mathrm{SOC}$ at the $\mathrm{Fe} / \mathrm{GaAs}$ interface, as has previously been predicted by first-principle calculations [15]. Our phenomenological model explains these anisotropies by the existence of interfacial Bychkov-Rashba and Dresselhaus spin-orbit fields and is able to reproduce the observed angular dependencies. 
Financial support by the Deutsche Forschungsgemeinschaft via Grants No. SFB 689 and No. GRK 1570 is gratefully acknowledged. P. H. acknowledges support from the International Doctorate Program, Topological Insulators of the Elite Network of Bavaria.

[1] J. Fabian, A. Matos-Abiague, C. Ertler, P. Stano, and I.Źutić, Acta Phys. Slovaca 57, 565 (2007).

[2] M. Gmitra, A. Matos-Abiague, C. Draxl, and J. Fabian, Phys. Rev. Lett. 111, 036603 (2013).

[3] J. Moser, A. Matos-Abiague, D. Schuh, W. Wegscheider, J. Fabian, and D. Weiss, Phys. Rev. Lett. 99, 056601 (2007).

[4] T. Uemura, Y. Imai, M. Harada, K.-i. Matsuda, and M. Yamamoto, Appl. Phys. Lett. 94, 182502 (2009).

[5] M. Wimmer, M. Lobenhofer, J. Moser, A. Matos-Abiague, D. Schuh, W. Wegscheider, J. Fabian, K. Richter, and D. Weiss, Phys. Rev. B 80, 121301 (2009).

[6] A. Matos-Abiague and J. Fabian, Phys. Rev. B 79, 155303 (2009).

[7] T. Hupfauer, A. Matos-Abiague, M. Gmitra, F. Schiller, J. Loher, D. Bougeard, C. H. Back, J. Fabian, and D. Weiss, Nat. Commun. 6, 7374 (2015).

[8] I. M. Miron, G. Gaudin, S. Auffret, B. Rodmacq, A. Schuhl, S. Pizzini, J. Vogel, and P. Gambardella, Nat. Mater. 9, 230 (2010).

[9] I. M. Miron, K. Garello, G. Gaudin, P.-J. Zermatten, M. V. Costache, S. Auffret, S. Bandiera, B. Rodmacq, A. Schuhl, and P. Gambardella, Nature (London) 476, 189 (2011).

[10] K. Garello, I. M. Miron, C. O. Avci, F. Freimuth, Y. Mokrousov, S. Blugel, S. Auffret, O. Boulle, G. Gaudin, and P. Gambardella, Nat. Nanotechnol. 8, 587 (2013).

[11] K.-S. Ryu, L. Thomas, S.-H. Yang, and S. Parkin, Nat. Nanotechnol. 8, 527 (2013).

[12] S. Emori, U. Bauer, S.-M. Ahn, E. Martinez, and G. S. D. Beach, Nat. Mater. 12, 611 (2013).
[13] S. Heinze, K. von Bergmann, M. Menzel, J. Brede, A. Kubetzka, R. Wiesendanger, G. Bihlmayer, and S. Blugel, Nat. Phys. 7, 713 (2011).

[14] S. Nadj-Perge, I. K. Drozdov, J. Li, H. Chen, S. Jeon, J. Seo, A. H. MacDonald, B. A. Bernevig, and A. Yazdani, Science 346, 602 (2014).

[15] S. Putz, M. Gmitra, and J. Fabian, Phys. Rev. B 90, 045315 (2014).

[16] Y. A. Bychkov and E. I. Rashba, JETP Lett. 39, 78 (1984).

[17] Y. A. Bychkov and E. I. Rashba, J. Phys. C 17, 6039 (1984).

[18] G. Dresselhaus, Phys. Rev. 100, 580 (1955).

[19] M. Trushin and J. Schliemann, Phys. Rev. B 75, 155323 (2007).

[20] See Supplemental Material at http://link.aps.org/ supplemental/10.1103/PhysRevLett.117.157202 for further details.

[21] M. Zölfl, M. Brockmann, M. Köhler, S. Kreuzer, T. Schweinböck, S. Miethaner, F. Bensch, and G. Bayreuther, J. Magn. Magn. Mater. 175, 16 (1997).

[22] M. Brockmann, M. Zölfl, S. Miethaner, and G. Bayreuther, J. Magn. Magn. Mater. 198-199, 384 (1999).

[23] F. Bensch, R. Moosbühler, and G. Bayreuther, J. Appl. Phys. 91, 8754 (2002).

[24] G. Bayreuther, M. Dumm, B. Uhl, R. Meier, and W. Kipferl, J. Appl. Phys. 93, 8230 (2003).

[25] M. Košuth, V. Popescu, H. Ebert, and G. Bayreuther, Europhys. Lett. 72, 816 (2005).

[26] K. Zakeri, T. Kebe, J. Lindner, and M. Farle, J. Magn. Magn. Mater. 299, L1 (2006).

[27] D. Weiss (private communication); M. Lobenhofer, Ph.D. Thesis, University Regensburg, 2013, http://epub .uni-regensburg.de/27633/1/Dissertation.pdf.

[28] A. N. Chantis, K. D. Belashchenko, E. Y. Tsymbal, and M. van Schilfgaarde, Phys. Rev. Lett. 98, 046601 (2007).

[29] H. Ebert, D. Ködderitzsch, and J. Minár, Rep. Prog. Phys. 74, 096501 (2011). 